MALNUTRICIÓN POR EXCESO EN LA EDAD ESCOLAR: ESTUDIO RETROSPECTIVO DE UNA COHORTE DE NIÑOS Y NIÑAS DE CIUDAD DE BUENOS AIRES (2012-2017)

\author{
MALNUTRITION BY EXCESS IN SCHOOLCHILDREN: \\ RETROSPECTIVE COHORT STUDY IN BUENOS AIRES CITY (2012-2017) \\ Piaggio Laura*, Schloss Beatriz*, Chiazzari Marisa*, Rolón Marina*, Mehlman Gustavo*, González \\ Rocío*. \\ *Ministerio de Salud de la Ciudad de Buenos Aires, Argentina. \\ Citation: Piaggio L., Schloss B., Chiazzari M., Rolón M., Mehlman G., González \\ R. (2020). Malnutrición por exceso en la edad escolar. Estudio retrospectivo \\ de una cohorte de niños y niñas de Ciudad de Buenos Aires (2012-2017). \\ Revista Salud Pública y Nutrición, 19 (4), 16-26. \\ Editor: Esteban G. Ramos Peña, Dr. CS., Universidad Autónoma de Nuevo \\ León, Facultad de Salud Pública y Nutrición, Monterrey Nuevo León, México. \\ Copyright: (C2020 Piaggio L., et al. This is an open-access article distributed \\ under the terms of Creative Commons Attribution License [CC BY 4.0], which \\ permits unrestricted use, distribution, and reproduction in any medium, \\ provided the original author and source are credited. \\ Competing interests: The authors have declared that no competing interests \\ exist. \\ DOI: $\underline{\text { https://doi.org/10.29105/respyn19.4-3 }}$ \\ Recibido: 04 de agosto 2020; \\ Aceptado: 07 de octubre 2020 \\ Email: antropologiaalimentaria@hotmail.com
}




\title{
MALNUTRICIÓN POR EXCESO EN LA EDAD ESCOLAR ESTUDIO RETROSPECTIVO DE UNA COHORTE DE NIÑOS Y NIÑAS DE CIUDAD DE BUENOS AIRES (2012-2017).
}

\author{
Piaggio Laura *, Schloss Beatriz *, Chiazzari Marisa *, Rolón Marina *, Mehlman Gustavo *, González Rocío *. \\ *Ministerio de Salud de la Ciudad de Buenos Aires, Argentina.
}

\begin{abstract}
RESUMEN
Introducción. La malnutrición por exceso es uno de los problemas de salud más prevalentes en escolares. Objetivo: describir el estado nutricional de una cohorte de niños/as que concurrieron a escuelas de gestión estatal de Ciudad de Buenos Aires, evaluados/as en 1ㅇ y 60 grado; analizar la prevalencia e incidencia de sobrepeso/obesidad y su asociación con sexo, zona, tipo de jornada escolar e intervenciones educativas del Programa Mi Escuela Saludable. Material y Método: estudio observacional, analítico, de cohorte retrospectivo. Se calculó el score z de IMC/edad y la diferencia de medias para muestras independientes. Se calcularon y compararon prevalencias mediante $\chi^{2}, p<0,05$. Se realizó el cálculo de regresión logística bivariada (OR IC95\%) para el análisis de incidencia. Resultados: Se incluyeron 3530 niños/as. La prevalencia de exceso de peso fue del 39,5\% en 2012 y del $48,3 \%$ en 2017. La prevalencia de obesidad aumentó significativamente en varones $(p=0,00)$ y en niños/as de escuelas de jornada simple sin intervención educativa $(p=0,01)$. La incidencia de obesidad tuvo una tasa de $3,1 \%$ y fue mayor en varones y en zona sur. Conclusiones: Durante la escolaridad primaria, se agrava la malnutrición por exceso, por lo que se requieren urgentes modificaciones del entorno alimentario escolar.

Palabras Clave: estado nutricional, salud escolar, obesidad.
\end{abstract}

\section{ABSTRACT}

Introduction: Malnutrition by excess is one of the most prevalent health issues in schoolchildren. Objective: To describe the nutritional status of a cohort of children attending public schools in Buenos Aires City, evaluated in 1st and 6th grade; to analyze the prevalence and incidence of overweight / obesity and their association with sex, geographic area, type of school day and educational interventions of the Program "My Healthy School". Material and method: Observational, analytical, retrospective cohort study. The BMI / age z score and the mean difference for independent samples were calculated. Prevalences were calculated and compared using $\chi^{2}, p<0.05$. The bivariate logistic regression was calculated (OR $\left.95 \% \mathrm{Cl}\right)$ for the incidence analysis. Results: 3530 children were included in the study. The prevalence of malnutrition by excess was $39,5 \%$ in 2012 and $48,3 \%$ in 2017 . The prevalence of obesity increased significantly in boys $(p=0,00)$ and in children from single-day schools without educational intervention $(p=0.01)$. The incidence of obesity had a rate of $3.1 \%$ and was higher in boys and in the southern area. Conclusions: During primary schooling, malnutrition by excess worsens, so urgent changes in the school food environment are needed.

Key words: Nutritional status, school health, obesity. 


\section{Introducción}

La malnutrición por exceso es uno de los problemas de salud más prevalentes en niños y niñas en edad escolar. En esta etapa se observa un marcado incremento en el sobrepeso y la obesidad. La Ciudad Autónoma de Buenos Aires (CABA) cuenta con información epidemiológica-nutricional sobre esta población procedente de diversas fuentes.

La Encuesta de Alimentación y Nutrición, que se realizó en la CABA en el año 2011, documentó en la población de niños y niñas de 5 a 12 años un $23,6 \%$ de sobrepeso y $18,8 \%$ de obesidad, siendo un $23,6 \%$ en los niños y un $13,8 \%$ en las niñas para esta última categoría (Vicejefatura de Gobierno de la Ciudad de Buenos Aires[VJ-GCBA], 2013). Un estudio epidemiológico en base a datos del Programa de Salud Escolar de la CABA del 2015 muestra para sobrepeso una prevalencia de $26,1 \%$ y para obesidad, un $20,6 \%$ en estudiantes de nivel primario (niños, $24,8 \%$ y niñas, $16,3 \%$ ) (Concilio, 2019). Asimismo, los datos del protocolo del Programa de Salud Escolar del año 2018 muestran entre la población de estudiantes de 6 a 14 años, un 21,8\% de sobrepeso y se verifica un $24,5 \%$ de obesidad (niños $28,9 \%$ y niñas, 18,9\%) (Ministerio de Salud Gobierno de la Ciudad de Buenos Aires[MS-GCBA], 2018a). Por último, otra fuente de datos de la CABA proviene del Informe de Población atendida en Centros de Salud de primer nivel de atención, del año 2018, que muestra que, para niños y niñas de 6 a 14 años, el sobrepeso fue del $24,6 \%$ y la obesidad del $20,9 \%$ (niños, 24,3\% y niñas, 17,7\%) (MS-GCBA, 2018b). A nivel nacional, según la 2a. Encuesta Nacional de Nutrición y Salud (ENNyS2) del 2019, la proporción de sobrepeso y obesidad fue del $20,7 \%$ y $20,4 \%$ respectivamente entre los 5 y los 17 años. En varones la obesidad fue de $22,9 \%$ y en mujeres $17,6 \%$ (Ministerio de Salud de la Nación[MSAL], 2019a). Otro estudio nacional de referencia es el del Programa de Sanidad Escolar (PROSANE) que publicó en el año 2019 una investigación sobre el estado nutricional de una cohorte de escolares con mediciones en los años 2012 y 2017. Sobre un total de 10961 estudiantes, en primer grado se observó una prevalencia de sobrepeso del $21,1 \%$ y una obesidad del $14,5 \%$, mientras que, en sexto grado, las prevalencias aumentaron a $26,6 \%$ de sobrepeso y $22,7 \%$ de obesidad (Lev, Coradini y González, 2019).
La obesidad genera gran preocupación sanitaria en la salud de niños y niñas por las consecuencias que genera: problemas de autoestima, afecciones respiratorias, articulares, hipertensión arterial y diabetes -a edades cada vez más tempranas. Asimismo, implica una mayor probabilidad de continuar con obesidad en la adultez, agravando sus consecuencias y el desarrollo de enfermedades no transmisibles (MSAL, 2018).

La escuela se destaca como un ámbito privilegiado para la promoción de prácticas saludables de alimentación, tanto por la importancia que tienen los alimentos y bebidas consumidos durante la jornada escolar, como por los procesos de socialización alimentaria que se desarrollan en las aulas, patios, comedores, salidas educativas, actos y festejos (Piaggio, et al. 2017; Piaggio, et al., 2011; 2013). Organismos internacionales han desarrollado múltiples iniciativas para aprovechar este potencial generador de salud de la institución escolar (Organización Mundial de la Salud[OMS], 2006; OMS, 2016).

A nivel local, el Programa de Salud Escolar (PSE) (Decreto $\left.\mathrm{N}^{\circ} 3362,1989\right)$ realiza controles de salud en las escuelas de gestión estatal, en $1^{\circ}, 4^{\circ}$ y $6^{\circ}$ grado $y$ en $2^{\circ}$ año de nivel medio.

La Ciudad cuenta con la Ley de Alimentación Saludable en Escuelas (Ley $\mathrm{N}^{\circ} 3704$, 2010) que plantea estándares nutricionales para los servicios de alimentación y establece el tipo de productos que se permite comercializar en los puntos de venta al interior de los establecimientos.

En el año 2012 se creó el Programa Mi Escuela Saludable (MES) con el objetivo de desarrollar acciones de educación alimentaria destinadas a escolares, docentes y familias. En las escuelas participantes, durante 2 años, Licenciados/as en Nutrición realizan talleres y se entrega bimestralmente un cuadernillo ("Mi Revista Saludable”) (González et al., 2015).

De las 443 escuelas primarias de gestión estatal, $40 \%$ son de jornada simple (JS) y $60 \%$ de jornada completa (JC) (Ministerio de Educación [MEGCBA], 2017). Todas brindan desayuno o merienda y, en las escuelas de JC, también se brinda almuerzo. 
Se distribuyen territorialmente en 21 distritos escolares (DE).

Retomando los antecedentes a nivel nacional, dado que el estudio del PROSANE no incluye a escolares de la CABA, se decidió analizar la base de datos jurisdiccional, a fin de comparar con los resultados de dicho estudio e indagar sobre algunos aspectos particulares de las escuelas de la Ciudad.

Se estableció como objetivo general describir el estado nutricional de una cohorte de estudiantes que concurrieron a escuelas de gestión estatal de la CABA, evaluados/as en $1^{\circ}$ y $6^{\circ}$ grado en los años 2012 y 2017, analizando la prevalencia e incidencia del exceso de peso.

Se planteó como hipótesis que durante la etapa escolar la prevalencia del exceso de peso se incrementa en la población estudiada, con mayor incidencia entre quienes concurren a escuelas ubicadas en la zona de mayor vulnerabilidad socioeconómica de la ciudad (zona sur).

\section{Material y Método}

El presente es un estudio observacional, analítico, de cohorte retrospectivo. Se tomaron las bases de datos del PSE para seleccionar una cohorte de estudiantes con mediciones antropométricas registradas en $1^{\circ} \mathrm{y}$ en $6^{\circ}$ grado. A la base se adicionó información sobre las escuelas que recibieron intervenciones educativas del Programa MES.

La población de estudio fue la totalidad de estudiantes que concurrieron a escuelas primarias de gestión estatal de la CABA evaluados/as por el PSE. Según datos oficiales (Ministerio de Educación[MEGCBA], 2019), la matrícula fue de 20630 estudiantes en $1^{\circ}$ grado en el año 2012 y de 20713 en $6^{\circ}$ grado en el 2017.

La cohorte se conformó retrospectivamente con aquellos escolares que contaban con datos de las mediciones antropométricas en el protocolo informatizado.

La base inicial fue de 3588 casos. Como criterio de inclusión se consideró a estudiantes con registro de las fechas de nacimiento y de la toma de las medidas antropométricas en $1^{\circ}$ y $6^{\circ}$ grado.
Los criterios de exclusión fueron los casos con datos faltantes de sexo y con mediciones extremas: \pm 5 desvíos estándar (DS) para Índice de Masa Corporal /Edad (IMC/E) (Organización Mundial de la Salud[OMS], 2009).

Luego de la revisión, la muestra quedó conformada por 3530 escolares.

La base incluyó las siguientes variables: fecha de nacimiento, sexo, fecha de las mediciones, peso y talla en 2012 y 2017 , nombre de la escuela a la que asistía en cada año, distrito escolar, zona de la ciudad, tipo de jornada escolar e intervención educativa del Programa MES.

Se utilizó el programa AnthroPlus de la Organización Mundial de la Salud (OMS, 2009) para el procesamiento de las mediciones antropométricas. Los valores de IMC/E se categorizaron de acuerdo a los criterios para evaluaciones poblacionales establecidas por el Ministerio de Salud de la Nación (Abeyá, et al., 2007):

- Bajo peso $=<-2,00$ Desvíos Standard (DS)

- Normal $=-2,00$ a $+0,99$ DS

- Sobrepeso $=+1,00 \mathrm{a}+2,00 \mathrm{DS}$

- Obesidad $=>+2,00$ DS

En cuanto a la distribución geográfica, se utilizó el criterio del Ministerio de Educación (ME-GCBA, 2019), que agrupa a los $21 \mathrm{DE}$ en 3 zonas: norte (DE 9, 10, 14, 15, 16, 17), centro (DE 1, 2, 3, 6, 7, 8, 11, $12,18)$ y sur (DE 4, 5, 13, 19, 20 y 21$)$.

Respecto a la jornada, las escuelas se clasificaron en dos tipos: simple y completa.

En relación a las intervenciones educativas, se consideraron dos categorías: presencia / no presencia del Programa MES en alguno de los años del período (2012-2016), considerando el ciclo lectivo completo. El análisis se organizó en dos fases: en la primera, se calcularon medidas de tendencia central y dispersión para variables cuantitativas (score $\mathrm{Z}$ de IMC/E) y se calculó la diferencia de medias para muestras independientes (2012 y 2017). Para variables categóricas (diagnósticos nutricionales al inicio y fin del período) se calcularon porcentajes y se compararon las prevalencias mediante chi cuadrado (x2). Se consideraron significativas las diferencias de $\mathrm{p}<0,05$. 
En la segunda fase, se analizó la incidencia del exceso de peso y, en particular, de la obesidad y su asociación con las variables dependientes (sexo, zona, tipo de jornada e intervención educativa). Para ello, se realizó un análisis multivariado con el cálculo de regresión logística bivariada, Odds Ratio (OR) con Intervalo de confianza (IC) $95 \%$.

El procesamiento estadístico se realizó con el programa IBM Statistical Package for Social Sciences (IBM SPSS Statistics 15.0).

En cuanto a las consideraciones éticas, para resguardar la identidad de niños y niñas y el secreto estadístico (Ley $\mathrm{N}^{\circ}$ 17622), luego de seleccionar los casos, el Área de Estadística del Ministerio de Salud de la CABA anonimizó la base de datos que fue posteriormente consolidada y procesada.

\section{Resultados}

Caracterización de la muestra

Se estudiaron 3530 escolares, 50,5\% masculino $(n=1782)$ y $49,5 \%$ femenino $(n=1748)$. En un $40 \%$ concurrían a escuelas ubicadas en la zona norte $(n=1402)$, un $43 \%$ en zona centro $(n=1509)$ y un $17 \%$ en zona sur $(\mathrm{n}=619)$. Esta distribución no guarda correlación con la matrícula escolar, que se distribuye $23 \%, 43 \%$ y $34 \%$ en las zonas norte, centro y sur, respectivamente. Un $54,3 \%$ de escolares $(n=1882)$ concurrieron a escuelas de JC, fluctuando levemente según zona de la Ciudad: $56,6 \%, 53,2 \%$ y $51,8 \%$ en el norte, centro y sur, respectivamente.

Un 34,3\% asistieron a escuelas en donde se desarrollaron actividades de educación alimentaria del Programa MES ( $\mathrm{n}=1212)$, correspondiendo $38,6 \%, 37,4 \%$ y $17,1 \%$ a las zonas norte, centro y sur. A la vez, el 73\% del alumnado $(\mathrm{n}=887)$ que participó en estas actividades correspondió a escuelas de JC.

\section{Comparación del estado nutricional en primer y sexto grado}

Respecto a la valoración del estado nutricional, al inicio del ciclo escolar, la media del score $\mathrm{Z}$ de IMC/E fue de 0,74 con un DS de $\pm 1,36$ (IC $95 \%=0,69-0,78)$. La mediana de IMC/E fue de 0,66. Al finalizar el período en estudio, la media de score $\mathrm{Z}$ de IMC/E fue de 0,88 con un DS de $\pm 1,21$ $($ IC95\% $=0,85-0,93)$. La mediana de IMC/E fue de 0,94 .
El score Z de IMC/E aumentó en promedio 0,15 $\pm 1,13$ entre 2012 y 2017 y esta diferencia fue estadísticamente significativa $(\mathrm{p}<0,001)$ (Test de $\mathrm{t}$ para datos pareados).

En el Gráfico 1 se observa la distribución de curvas de la muestra total y según sexo. Tanto al inicio como al final, la curva de la muestra total se encuentra desplazada a la derecha, desplazamiento más acentuado en varones. Esto refleja valores más altos del score $\mathrm{Z}$ de IMC/E, lo cual indica mayor frecuencia de sobrepeso y obesidad.

Gráfico 1 - Comparación de la población estudiada con la distribución de referencia. Muestra total y según sexo. Años 2012 y 2017.
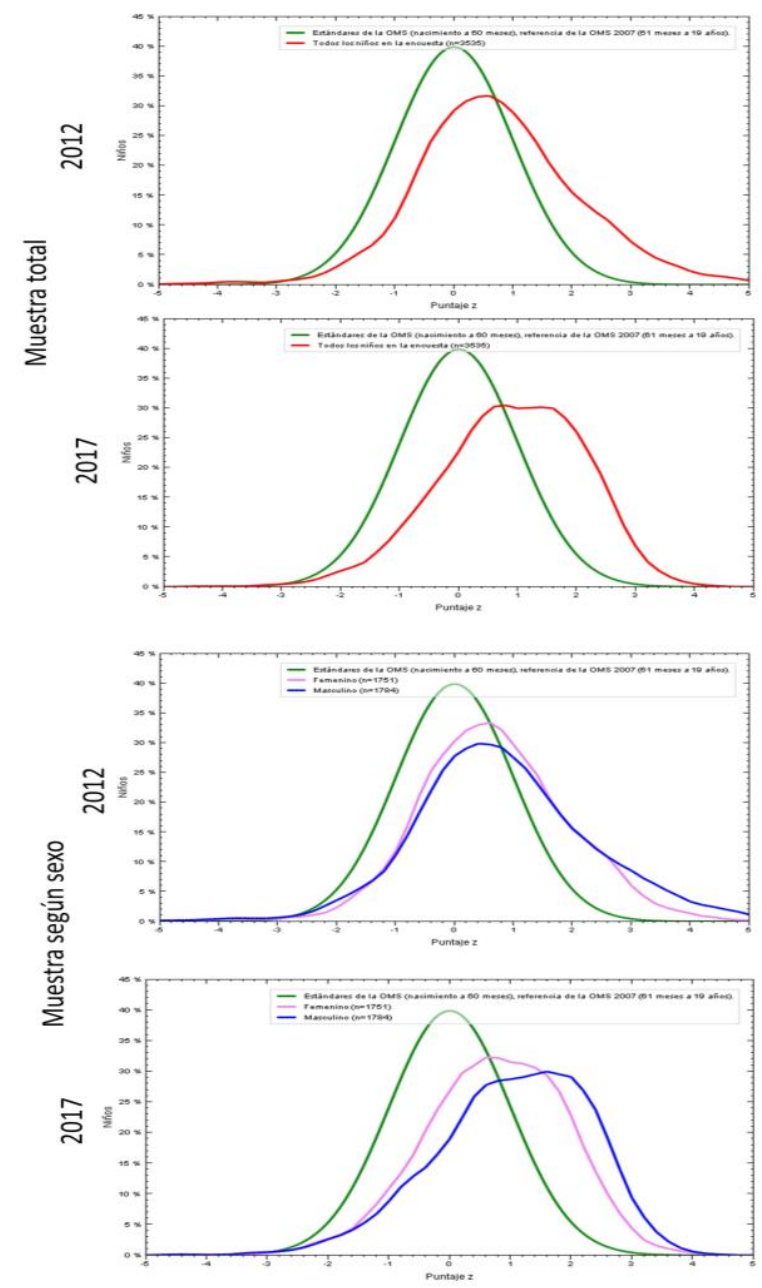
Al inicio del ciclo escolar, la prevalencia de sobrepeso fue $22,6 \%$ y de obesidad $16,9 \%$. Hacia el final del estudio, las prevalencias aumentaron a $29,5 \%$ de sobrepeso y $18,8 \%$ de obesidad (Tabla 1 ).

Tabla 1 - Distribución porcentual de los diagnósticos del estado

\begin{tabular}{lcccc}
\hline \multirow{2}{*}{ Diagnóstico } & \multicolumn{2}{c}{2012} & \multicolumn{2}{c}{2017} \\
\cline { 2 - 5 } Bajo peso & $\mathrm{n}$ & $\% \mathrm{IC} \mathrm{I}_{95}$ & $\mathrm{n}$ & $\% \mathrm{IC} \mathrm{C}_{95}$ \\
& & 1.7 & 50 & 1.4 \\
Normo-peso & 2072 & $(1,33-2,22)$ & & $(1,05-1,86)$ \\
& & $(57,25-60,33)$ & 1776 & 50.3 \\
Sobrepeso & 799 & 22.6 & & $(40,65-51,97)$ \\
& & $(21,26-24,05)$ & 1040 & 29.5 \\
Obesidad & 598 & 16.9 & & $(27,96-30,99)$ \\
Total & 3530 & $(15,72-18,22)$ & 664 & 18.8 \\
\hline
\end{tabular}

Fuente: elaboración propia en base a datos del PSE IC $95 \%$

La prevalencia de exceso de peso (sobrepeso + obesidad) fue de $39,5 \%$ en 2012 y de 48,3\% en 2017, incrementándose a lo largo de 6 años de escolaridad en un $22 \%$.

Analizando las variables en estudio (Tabla 2), el sobrepeso se incrementó de manera significativa en ambos sexos $(p<0,000)$. La obesidad se incrementó significativamente sólo en varones $(\mathrm{p}<0,000)$. Al final del periodo, la prevalencia de obesidad entre varones casi duplica a la de mujeres.

En las zonas norte y centro se incrementó significativamente el sobrepeso $(p<0,000)$ y, la obesidad sólo en la zona sur $(\mathrm{p}=0,009)$. Al observar las prevalencias de obesidad por zona en el final del período, la misma fue de $16,6 \%$ en zona norte y $23,9 \%$ en el sur, siendo la diferencia entre ambas zonas de un $44 \%$.

En relación al tipo de jornada escolar, el sobrepeso se incrementó significativamente en JS y JC $(\mathrm{p}=0,002$ y $\mathrm{p}<0,000)$, pero la obesidad aumentó sólo en JS $(\mathrm{p}=0,01)$.

Un comportamiento similar se observó entre escolares que asistieron a escuelas con presencia del Programa MES. El sobrepeso se incrementó tanto en escuelas con y sin intervención $(\mathrm{p}<0,000)$, pero la obesidad aumentó sólo en éstas últimas $(\mathrm{p}=0,005)$.

\begin{tabular}{|c|c|c|c|c|c|c|c|}
\hline \multirow{3}{*}{ Variable } & \multirow{3}{*}{ Categorías } & \multicolumn{6}{|c|}{ Categorías de exceso de peso } \\
\hline & & \multicolumn{3}{|c|}{ Sobrepeso } & \multicolumn{3}{|c|}{ Obesidad } \\
\hline & & 2012 & 2017 & $p$ & 2012 & 2017 & $p$ \\
\hline \multirow{3}{*}{ Sexo } & $F(n=1748)$ & $23.30 \%$ & $29.60 \%$ & 0.000 & $14.50 \%$ & $13.20 \%$ & 0.28 \\
\hline & $M(n=1782)$ & $21.90 \%$ & $29.30 \%$ & 0.000 & $19.40 \%$ & $24.30 \%$ & 0.000 \\
\hline & & & & & 0.000 & 0.000 & \\
\hline \multirow{4}{*}{ Zona Ciudad } & Norte $(n=1402)$ & $21.10 \%$ & $28.60 \%$ & 0.000 & $17.10 \%$ & $16.60 \%$ & 0.72 \\
\hline & Centro $(n=1509)$ & $23.00 \%$ & $30.20 \%$ & 0.000 & $16.40 \%$ & $18.80 \%$ & 0.08 \\
\hline & Sur $(n=619)$ & $25.20 \%$ & $29.60 \%$ & 0.09 & $17.90 \%$ & $23.90 \%$ & 0.01 \\
\hline & & & & & & 0.000 & \\
\hline \multirow{3}{*}{ Tipo de Jornada* } & Simple $(n=1583)$ & $22.60 \%$ & $27.20 \%$ & 0 & $16.70 \%$ & $20.20 \%$ & 0.01 \\
\hline & Completa ( $n=1882$ ) & $22.70 \%$ & $31.40 \%$ & 0.000 & $17.20 \%$ & $17.80 \%$ & 0.64 \\
\hline & & & 0.007 & & & & \\
\hline \multirow{3}{*}{ Intervención MES } & $\operatorname{Sin}(n=2318)$ & $22.60 \%$ & $29.00 \%$ & 0.000 & $15.60 \%$ & $18.70 \%$ & 0.01 \\
\hline & Con $(n=1212)$ & $22.70 \%$ & $30.30 \%$ & 0.000 & $19.50 \%$ & $19.10 \%$ & 0.8 \\
\hline & & & & & 0.003 & & \\
\hline
\end{tabular}

Nota: * se excluyen 65 casos que cambiaron de jornada en el período considerado. Fuente: elaboración propia en base a datos del PSE y del Programa MES

Comparando las prevalencias de sobrepeso y obesidad en 2012 y 2017 por tipo de jornada combinada con la presencia del Programa MES (Tabla 3), el sobrepeso se incrementó en todos los casos, pero la obesidad aumentó significativamente sólo entre estudiantes que concurrieron a escuelas de JS sin intervención del Programa MES ( $\mathrm{p}=0,01)$.

Tabla 3 - Distribución porcentual del exceso de peso por tipo de jornada e intervención educativa combinadas, en 2012 y 2017

\begin{tabular}{|c|c|c|c|c|}
\hline \multirow{3}{*}{$\begin{array}{l}\text { Tipo de jornada* }{ }^{*} \\
\text { intervención educativa } \\
\text { combinadas }\end{array}$} & \multicolumn{4}{|c|}{ Categorías de exceso de peso } \\
\hline & \multicolumn{2}{|c|}{ Sobrepeso } & \multicolumn{2}{|c|}{ Obesidad } \\
\hline & 2012 & 2017 & 2012 & 2017 \\
\hline $\begin{array}{l}\text { JC con interv MES } \\
(n=887)\end{array}$ & $22.40 \%$ & $30.80 \%$ & $20.00 \%$ & $19.20 \%$ \\
\hline $\begin{array}{l}\text { JC sin interv MES } \\
(n=995)\end{array}$ & $23.00 \%$ & $32.00 \%$ & $14.80 \%$ & $16.60 \%$ \\
\hline $\begin{array}{l}\text { JS con interv MES } \\
(n=303)\end{array}$ & $24.80 \%$ & $29.00 \%$ & $17.50 \%$ & $19.50 \%$ \\
\hline $\begin{array}{l}\text { JS sin interv MES }{ }^{1,2} \\
(n=1280)\end{array}$ & $22.00 \%$ & $26.70 \%$ & $16.50 \%$ & $20.40 \%$ \\
\hline
\end{tabular}

Nota: * se excluyen 65 casos que cambiaron de jornada en el período considerado Fuente: elaboración propia en base a datos del PSE y del Programa MES 1. $p<0.01$ Sobrepeso

2. $p<0.05$ Obesidad

Evolución del estado nutricional

Tomando en consideración las categorías de diagnóstico nutricional al inicio del ciclo escolar, transcurridos seis años, las mismas presentaron las siguientes variaciones:

- De 61 escolares con bajo peso al inicio, el 14,8\% llegó a $6^{\circ}$ grado con sobrepeso u obesidad.

- De 2072 escolares con normopeso al inicio, el 69\% continuaba como normal. El $23,8 \%$ cambió a 
sobrepeso y el 5,3\% a obesidad, finalizando con exceso de peso el $29,1 \%$.

- De 799 escolares con sobrepeso inicial, el 29\% cambió a normopeso, el 46,1\% continuó con sobrepeso y el $25 \%$ cambió a obesidad; finalizando con exceso de peso el $71,1 \%$.

- De 598 escolares con obesidad al inicio, el 59\% continuaba con obesidad y el $28,6 \%$ finalizó con sobrepeso, sumando $77,6 \%$ con exceso de peso. Un $12 \%$ cambió a normopeso.

En el conjunto de la muestra, 812 escolares empeoraron su diagnóstico nutricional (23\%), considerando tanto quienes migraron a "exceso de peso" ( $\mathrm{n}=612)$ como quienes de sobrepeso pasaron a obesidad $(n=200)$. Sin embargo, también podemos observar que 476 mejoraron su estado nutricional $(13,5 \%)$, pasando 171 de obesidad a sobrepeso y 305 de ambas categorías a "sin exceso".

A continuación, se presentan los datos de acuerdo a las cuatro categorías utilizadas en el Informe del PROSANE (Lev, Coradini y González, 2019) (Gráfico 2).

Gráfico 2 - Distribución de la población según estado nutrición agrupado (CON / SIN sobrepeso/obesidad) entre los años 2012 y 2017.

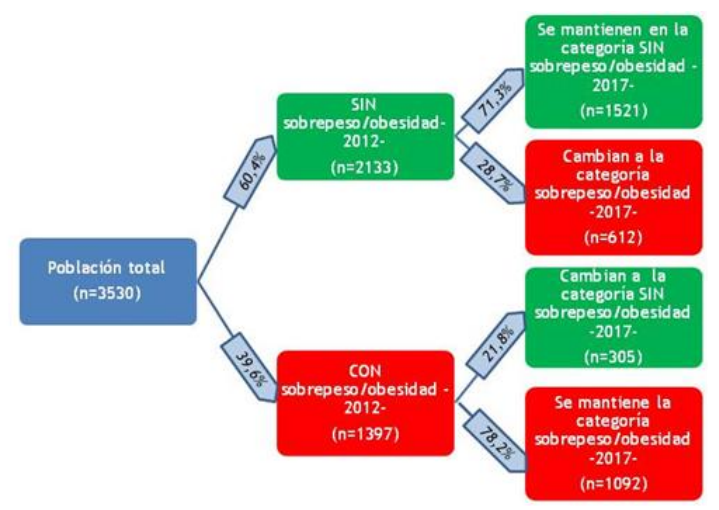

Fuente: elaboración propia en base a datos del Programa de Salud Escolar (MS-GCBA).

Relacionando estas categorías con las variables en estudio, sólo se observan diferencias significativas según zona de la ciudad, siendo mayor el porcentaje de estudiantes que se mantienen sin exceso de peso en las escuelas de zona norte $(\mathrm{p}=0,022)$, mientras que en la zona sur es mayor el porcentaje de quienes continúan con exceso de peso $(\mathrm{p}=0,006)$.

Se realizó un análisis multivariado focalizando entre quienes migraron desde otras categorías hacia el exceso de peso y, en particular, hacia la obesidad, considerando como variables dependientes: sexo, zona de la ciudad dicotomizada (sur / no-sur), tipo de jornada e intervención del Programa MES.

Respecto a la incidencia de exceso de peso, cuya tasa fue de $17,3 \%$, la regresión logística bivariada sólo evidenció asociación para la variable sexo: los varones tuvieron un $48 \%$ más probabilidad de cambiar a dicha categoría ( $<<0,000$; OR: 1,488; IC95\%: 1,245-1,778).

La tasa de incidencia de obesidad fue de $3,1 \%$. Se evidenció asociación para las variables sexo y zona: los varones duplicaron la probabilidad de cambiar a dicha categoría ( $\mathrm{p}<0,000$; OR:2,331; IC95\%:1,812$2,999)$ y quienes concurrieron a escuelas de zona sur tuvieron un $80 \%$ más probabilidad $(\mathrm{p}=0,005$; OR:1,793; IC95\%:1,190-2,702).

Sintetizando destacamos que, en el análisis de comparación de prevalencias de obesidad, la misma se incrementó significativamente por sexo (varones), por zona (sur) y en estudiantes que concurrieron a escuelas de JS sin intervención del Programa MES. Al analizar la incidencia de obesidad, resultaron asociadas sólo las variables sexo y zona.

\section{Discusión}

En este estudio se documentó un incremento significativo del exceso de peso durante los seis años de escolaridad primaria (2012-2017). Casi la mitad de estudiantes de escuelas primarias de gestión estatal de la CABA $(48,3 \%)$ se encontraron con exceso de peso al final del período considerado, similar a lo evaluado a nivel nacional por el PROSANE (49,3\%) (Lev, Coradini y González, 2019).

El sobrepeso afectó casi por igual a ambos sexos, pero la obesidad resultó mayor en varones. Esta diferencia por sexo también se documentó en los estudios locales citados como antecedentes (Concilio, 2019; GCBA, 2013; GCBA, 2018a, 2018b) y en la Segunda Encuesta Nacional de 
Nutrición y Salud (MSAL, 2019a), que además identificó diferencias significativas según sexo en algunos consumos alimentarios: menor consumo de frutas y mayor consumo de bebidas azucaradas en varones.

La mayor prevalencia de obesidad en varones, según el Atlas Global de Obesidad infantil (Lobstein y Brindsen, 2019), es una tendencia que se observa en los países de altos y medianos-altos ingresos (entre los que se encuentra Argentina) tanto en el grupo de edad de 5 a 9 años como en el de 10 a 19. Sha, et al. (2020) plantean que ha habido escaso debate acerca de las posibles razones e implicancias de estas diferencias. Instan a incorporar una perspectiva de género en los estudios de obesidad infantil para contribuir a mejorar las acciones de prevención.

Respecto a la distribución geográfica, en nuestra muestra se observó que el incremento en la prevalencia e incidencia de obesidad fue significativamente mayor entre el alumnado que concurrió a escuelas de la zona sur de la ciudad. Esta zona se caracteriza por una alta concentración de población en situación de vulnerabilidad social (Abelenda, Canevari y Montes, 2016), indicando que la obesidad en la infancia se presenta con mayor intensidad en contextos de pobreza. Esta asociación se verificó en otros estudios de escolares que concurren a escuelas de gestión estatal (Concilio, 2019; Ponce, et al., 2016), no así en población infantil general (VJ-GCBA, 2013; MSAL, 2019a).

Considerando el tipo de jornada escolar, la prevalencia de obesidad se incrementó significativamente en las escuelas de jornada simple. Diversos documentos de organismos internacionales y nacionales (Castagnari, 2018; MSAL, 2019b; Organización Panamericana de la Salud[OPS], 2014; United Nations International Children's Emergency Fund[UNICEF]-Fundación InterAmericana del Corazón[FIC] 2018; OMS, 2016) destacan la provisión de comidas de adecuada calidad nutricional en la escuela como una medida estratégica para abordar la obesidad infantil.

Una investigación desarrollada unos años antes del período considerado (Piaggio et al, 2011) mostró una relación inversa entre los consumos informales durante los recreos y las comidas estructuradas brindadas por la escuela: el consumo de bebidas azucaradas, golosinas, alfajores, galletitas, snacks salados era mayor en escuelas de JS que en las de JC (en las que se ofrece desayuno y almuerzo). En este sentido, el mayor consumo informal de productos de inadecuado perfil nutricional en escuelas de JS, podría estar relacionado con el incremento significativo de la obesidad en este tipo de jornada. No obstante, ello requeriría futuras investigaciones ya que el presente estudio no ha contado con información sobre ingesta alimentaria y, por tanto, tiene como limitación el no poder establecer asociaciones directas entre el estado nutricional y la alimentación escolar.

Las intervenciones de educación alimentaria del Programa MES se desarrollaron en mayor proporción en escuelas de JC durante el período considerado. Podemos postular que dichas intervenciones junto con este tipo de jornada ejercieron un efecto sinérgico en la contención del incremento de la obesidad.

La bibliografía indica que las intervenciones educativas tienen una efectividad limitada en la mejora de las prácticas alimentarias en la infancia y de su estado nutricional, cuando no se dan acompañadas por modificaciones en el entorno escolar (Ávila, et al. 2016; Hawkes, 2013; Rossi et al., 2018; UNICEF-FIC, 2018). En este sentido, se han documentado cambios favorables en las preferencias alimentarias y prácticas de consumo de escolares cuando las intervenciones educativas se han dado, por ejemplo, en el marco de experiencias sostenidas de entrega gratuita de frutas o de incremento de la disponibilidad de agua segura sumada a la restricción de bebidas azucaradas (World Cancer Research Fund[WCRF], 2015).

Una evaluación propia del programa MES concluyó que "para que la educación alimentaria-nutricional sea traducida en acciones, se requieren decisiones políticas que vayan en línea con cambios estructurales en el ambiente para que el entorno realmente favorezca los hábitos saludables" (Rossi et al., 2018, p.45).

Se cuenta con evidencia a nivel internacional que muestra que las modificaciones en los entornos alimentarios escolares han producido efectos positivos en la mejora de las prácticas alimentarias infantiles y en una disminución del IMC (Driessen, Cameron, Thornton, Lai y Barnett, 2014). Una herramienta clave la constituyen las regulaciones 
mediante leyes estrictas con estándares claros. Un estudio realizado en EEUU (Taber, Chriqui, Perna, Powell y Chaloupka, 2012) analizó el efecto de diferentes leyes dictadas en 40 Estados para regular la venta de los llamados "alimentos competitivos" (aquellos que se venden por fuera de las comidas provistas por la escuela). Observaron un menor incremento del IMC, luego de 3 años, en estudiantes expuestos a entornos escolares regulados por leyes fuertes (que establecían estándares claros y específicos y tenían consistencia a lo largo de los niveles escolares), en comparación con estudiantes de Estados con regulaciones débiles, entre quienes el incremento de IMC fue similar al de estudiantes de Estados sin ninguna normativa.

En relación a los puntos de venta de alimentos y bebidas, la reglamentación de la Ley de Alimentación Saludable de la CABA (Ley N ${ }^{\circ}$ 3704, 2010) sólo exige que se agreguen productos saludables a la oferta convencional, lo cual la sitúa en la categoría de "regulación débil". En los estándares nacionales e internacionales (MSAL, 2019b; UNICEF-FIC, 2018; OMS, 2016) se plantea que la oferta de alimentos y bebidas en el ámbito escolar (disponible para la venta, traída por estudiantes, ofrecida en eventos festivos, deportivos o salidas educativas) debe ser exclusivamente de buena calidad nutricional, priorizando alimentos naturales o mínimamente procesados, evitando la presencia de productos ultraprocesados (Castagnari, 2018) y asegurando la disponibilidad gratuita de agua segura. Asimismo, es fundamental incluir la restricción de toda publicidad de alimentos y bebidas, marcas y empresas, a fin de ofrecer un entorno escolar protegido (MSAL, 2019b; OPS, 2014; OMS, 2016).

A la vez, el impacto diferencial de la obesidad en la población que concurre a escuelas ubicadas en la zona de mayor vulnerabilidad socio-económica de la ciudad (zona sur) podría aminorarse incrementando la oferta de escuelas de jornada completa (Asociación Civil por la Igualdad y la Justicia[ACIJ], 2012). La desigual distribución geográfica de este tipo de oferta educativa no se subsana con la propuesta de jornada extendida (sumar dos horas de clase y almuerzo a la jornada simple), iniciada en 2017 (Di Pietro, Tófalo, Medela, Pitton, y Zanelli, 2017), ya que la misma no abarca al total de la matrícula (se implementa sólo en los últimos dos años de escolaridad). Incrementar las escuelas de jornada completa en el sur de la ciudad permitiría mejorar la seguridad alimentaria de niños y niñas.

\section{Conclusiones}

La malnutrición por exceso afecta a la mitad de los y las estudiantes de escuelas primarias de gestión estatal de la CABA. Durante el período escolar, un alto porcentaje de niños y niñas empeora su estado nutricional, habiéndose documentado una mayor incidencia de obesidad en varones y en la zona de más vulnerabilidad socio-económica de la ciudad.

Se considera importante desarrollar líneas de investigación que analicen la mayor prevalencia de obesidad en los varones incorporando una perspectiva de género.

Por otro lado, se plantea necesario ampliar la oferta educativa de jornada completa, para contribuir -entre otros aspectos- a mejorar la seguridad alimentaria de niños y niñas de la zona sur.

Autoridades sanitarias internacionales y nacionales han destacado la potencialidad del entorno escolar en la prevención de la obesidad infantil. Transformar en acto esa potencialidad en la Ciudad de Buenos Aires requiere implementar con urgencia modificaciones en el entorno escolar a través de regulaciones estrictas, basadas en evidencia científica y $\sin$ conflictos de interés.

\section{Bibliografía}

Abelenda, N., Canevari, J. y Montes, N. (2016). Territorios de mayor vulnerabilidad social en la Ciudad Autónoma de Buenos Aires. Recorrido en perspectiva histórica sobre aspectos estructurales no resueltos. Revista Población de Buenos Aires, 13(23), 7-30. Recuperado de: https://www.redalyc.org/articulo.oa?id=7404559 2001

Abeyá-Gilardón, E, Calvo, E., Durán, P. Longo, E., Mazza, C. (2007). Evaluación del estado nutricional de niñas, niños y embarazadas mediante antropometría. Argentina. Ministerio de Salud de la Nación. Recuperado de: http://www.msal.gob.ar/images/stories/bes/grafi cos/0000000256cnt-a07-manual-evaluacionnutricional.pdf 
Asociación Civil por la Igualdad y la Justicia [ACIJ] (2012). La distribución discriminatoria de la educación primaria de jornada completa en la Ciudad de Buenos Aires. Recuperado de https://acij.org.ar/wp-

content/uploads/2012/12/Jornada-completa-

compilado.pdf

Ávila, M., Huertas, F., y Tercedor, P. (2016). Programas de intervención para la promoción de hábitos alimentarios y actividad física en escolares españoles de Educación Primaria: revisión sistemática. Nutrición Hospitalaria, 33(6), 1438-1443.

doi: https://doi.org/10.20960/nh.807

Castagnari, V. (2019). Obesidad: una cuestión de derechos de niños, niñas y adolescentes. UNICEF, Argentina.

https://www.unicef.org/argentina/media/4996/fil e/Obesidad,\%20una\%20cuesti\%C3\%B3n\%20de $\%$ 20derechos.pdf

Concilio, M. (2019). Estudio transversal sobre el estado nutricional de la población bajo programa de Salud Escolar en la Ciudad Autónoma de Buenos Aires en el año 2015. (Tesis de maestría). Universidad Nacional de Lanús. Buenos Aires. Argentina

Decreto $N^{\circ}$ 3362. (1989). Boletín Oficial. Buenos Aires, Argentina. Programa Salud Escolar. Recuperado de: https://boletinoficial.buenosaires.gob.ar/normati vaba/norma/27359

Di Pietro S., Tófalo, A., Medela, P., Pitton, E., Zanelli, M. (2017). El proyecto de Jornada Extendida en su primer año de implementación Estudio sobre sus propósitos, avances y desafios. Unidad de Evaluación Integral de la Calidad y Equidad Educativa del Ministerio de Educación del Gobierno de la Ciudad de Buenos Aires. Recuperado de: https://www.buenosaires.gob.ar/sites/gcaba/files /informe_de_investigacion__jornada_extendida.pdf

Driessen, C., Cameron, A., Thornton, L., Lai, S., Barnett, L. (2014). Effect of changes to the school food environment on eating behaviours and/or body weight in children: A systematic review. Obesity Reviews, 15(12), 968-982. doi: https://doi.org/10.1111/obr.12224

González, V., Antún, M., Casagrande, M., Di Paola, M. Eisenberg, M., Escasany, M., Yomal A. (2015). Experiencia del Programa Mi Escuela Saludable. Actualización En Nutrición, 16, pp.51-61.

Hawkes, C. (2013). Promoting healthy diets to prevent and control obesity and diet-related chronic disease. Food and Agriculture Organization of the United Nation[FAO]. Recuperado http://www.fao.org/3/i3235e/i3235e.pdf

Lev, D.; Coradini, G.; González, P. (2019). Valoración antropométrica al inicio y al finalizar el ciclo de educación primaria en Argentina. Programa de Sanidad Escolar[PROSANE]. Recuperado de: http://www.msal.gob.ar/images/stories/bes/grafi cos/0000001405cnt-Valoracin-antropomtricainicio-y-al-finalizar-ciclo-educacin-primariaArgentina-07-03-2019.pdf

Ley $\mathrm{N}^{\circ}$ 3704. (2010). Boletín Oficial. Buenos Aires, Argentina. Alimentación saludable de niños, niñas y adolescentes en edad escolar. Recuperado de: http://www2.cedom.gob.ar/es/legislacion/norma s/leyes/anexos/drl3704.html

Lobstein, T., Brinsden H. (2019) Atlas of childhood obesity. World Obesity Federation. Recuperado de: http://s3-eu-west-1.amazonaws.com/woffiles/11996_Childhood_Obesity_Atlas_Report_ ART_V2.pdf

Ministerio de Educación. Gobierno de la Ciudad Autónoma de Buenos Aires[ME-GCBA]. (2019). Matrícula, unidades educativas y secciones. Serie histórica 1996/2019. Recuperado de: https://www.buenosaires.gob.ar/calidadyequidad educativa/estadistica/series-historicas

Ministerio de Educación. Gobierno de la Ciudad Autónoma de Buenos Aires[ME-GCBA]. (2017). Establecimientos educativos sector estatal. Recuperado 
https://www.buenosaires.gob.ar/sites/gcaba/files /ueicee_registroestablecimientos-estatal-201704-30.pdf

Ministerio de Educación. Gobierno de la Ciudad Autónoma de Buenos Aires[ME-GCBA]. (2019). Educación Común. Nivel Primario. Anuario Estadístico. Recuperado de: https://www.buenosaires.gob.ar/calidadyequidad educativa/estadistica/anuario/educacion-comunnivel-primario

Ministerio de Salud-Gobierno de la Ciudad de Buenos Aires[MS-GCBA]. (2018a). Programa Salud Escolar. Protocolo del control de salud en las escuelas. Recuperado de: http://bit.ly/saludescolar2017

Ministerio de Salud-Gobierno de la Ciudad de Buenos Aires[MS-GCBA]. (2018b). Informe de la situación nutricional de la población atendida en Centros de Salud Nivel 1. Recuperado de: https://www.buenosaires.gob.ar/sites/gcaba/files /informe_progrnutric_datos_cesacs_2017.pdf

Ministerio de Salud de la Nación[MSAL] (2018). Sobrepeso y obesidad en niños, niñas $y$ adolescentes según datos del primer nivel de atención en la Argentina. Recuperado de: https://www.unicef.org/argentina/media/4221/fil e/Obesidad Infantil.pdf

Ministerio de Salud de la Nación[MSAL] (2019a). 2da. Encuesta Nacional de Nutrición y Salud. Indicadores priorizados. Recuperado de: http://www.msal.gob.ar/images/stories/bes/grafi cos/0000001602cnt-2019-10_encuesta-nacionalde-nutricion-y-salud.pdf

Ministerio de Salud de la Nación[MSAL] (2019b). Guía de Entornos Escolares Saludables. Recuperado de: http://www.msal.gob.ar/images/stories/bes/grafi cos/0000001439cnt-2019-06_entornosescolares-saludables.pdf

Organización Mundial de la Salud[OMS]. (2006). Development of a framework on the Nutritionfriendly Schools Initiative (Issue February). Recuperado
http://www.who.int/nutrition/publications/Montr eux_Meeting_Report.pdf

Organización Mundial de la Salud[OMS]. (2009). WHO AnthroPlus for Personal Computers Manual: Software for assessing growth of the world's children and adolescents. Recuperado de: http://www.who.int/growthref/tools/en/

Organización Mundial de la Salud[OMS]. (2016). Informe de la comisión para acabar con la obesidad infantil. doi: https://doi.org/ISBN 978 9243510064

Organización Panamericana de la Salud[OPS]. (2014). Plan de acción para la prevención de la obesidad en la niñez y la adolescencia. Recuperado de: https://www.paho.org/hq/dmdocuments/2015/O besity-Plan-Of-Action-Child-Spa-2015.pdf

Piaggio, L., Concilio, M., Rolón, M., Macedra, G., Dupraz, S. (2011). Alimentación infantil en el ámbito escolar: entre patios, aulas y comedores. Salud Colectiva, 7(2), pp.199-213. Recuperado de:

http://revistas.unla.edu.ar/saludcolectiva/article/ view/380/pdf_57

Piaggio, L., Concilio, M., Rolón, M., Macedra, G., Dupraz, S. (2013). Alimentación escolar: ¿asistencia o educación? Espacio, tiempo y organización del cuidado infantil. Revista Novedades Educativas, 274, pp.1-11.

Piaggio, L., Duarte, M., Montenegro, B., Peri, C., Schloss, B., Rolón, M. (2017). Educación alimentaria: complejidad e interdisciplina en una guía digital. Recuperado de: http://bit.ly/caespo2017

Ponce, M., Allemandi, L., Castronuovo, L. Tiscornia, V., Gukowski, P., Schoj, V. (2016). Brechas sociales de la obesidad en la niñez y adolescencia. Análisis de la Encuesta Mundial de Salud Escolar. UNICEF Argentina. Recuperado de:

https://www.unicef.org/argentina/spanish/Salud_ U-InformeObesidad2016ok.pdf 
Rossi, M., Antún, M., y Casagrande, M., Escasany, M., Ferrari, M., Raele, G. (2018). Evaluación de la intervención del programa mi escuela saludable en una cohorte de escuelas que participaron durante 2016-2017. Revista de La Facultad de Ciencias Médicas, 76(1), pp. 37-46. doi:https://doi.org/10.31053/1853.0605.v76.n1.2 2574

Shah, B., Tombeau Cost, K., Fuller, A., Birken, C., Anderson, L. (2020) Sex and gender differences in childhood obesity: contributing to the research agenda. BMJ Nutrition, Prevention \& Health: p.1-3. Recuperado de: https://nutrition.bmj.com/content/early/2020/09/ 07/bmjnph-2020-000074

Taber, D., Chriqui, J., Perna, F., Powell, L., Chaloupka, F. (2012). Weight status among adolescents in states that govern competitive food nutrition content. Pediatrics, 130(3), 437-444. doi: https://doi.org/10.1542/peds.2011-3353

United Nations International Children's Emergency Fund[UNICEF], Fundación InterAmericana del Corazón [FIC]. (2018). Entornos Escolares Saludables. Recomendaciones para promover políticas escolares que prevengan la obesidad infantil en la Argentina. Coalición Nacional para prevenir la obesidad en niños, niñas y adolescentes. Recuperado de: https://www.unicef.org/argentina/sites/unicef.or g.argentina/files/2018-

11/SALUD_1811_entornos_escolares.pdf

Vicejefatura de Gobierno de la Ciudad de Buenos Aires [VJ-GCBA]. (2013). Primera Encuesta Alimentaria y Nutricional de la Ciudad Autónoma de Buenos Aires. EAN-CABA 2011. Documento de Resultados, vol (1). Buenos Aires, Argentina

World Cancer Research Fund[WCRF]. (2015). Curbing global sugar consumption: Effective food policy actions to help promote healthy diets and tackle obesity. Recuperado de: http://www.wcrf.org/sites/default/files/CurbingGlobal-Sugar-Consumption.pdf. 